\title{
LA FUNCIÓN DE SEGURIDAD EN LA LEGALIDAD CONSTITUCIONAL
}

\author{
ALESSANDRO PACE
}


SUMARIO

I. EL POR QUÉ ES INEXACTO Y ENGAÑOSO HABLAR DE UN DERECHO A LA SEGURIDAD. II. SOBRE QUÉ DEBE ENTENDERSE POR SEGURIDAD EN RELACIÓN CON EL ORDEN PÚBLICO. III. LOS LÍMITES IMPUESTOS A LA FUNCIÓN DE SEGURIDAD DE LA LEGALIDAD CONSTITUCIONAL. 


\title{
LA FUNCIÓN DE SEGURIDAD EN LA LEGALIDAD CONSTITUCIONAL
}

\author{
ALESSANDRO PACE \\ Catedrático Emérito de Derecho Constitucional \\ Universidad «La Sapienza» de Roma.
}

\section{EL POR QUÉ ES INEXACTO Y ENGAÑOSO HABLAR DE UN DERECHO A LA SEGURIDAD}

Resulta indiscutible que, tras los acontecimientos — que, por desgracia, no han permanecido aislados- del 11 de septiembre de 2011, la percepción de la seguridad pública ha cambiado profundamente ${ }^{1}$. Sin embargo, ello no justifica la tesis según la cual la difusa necesidad de la seguridad habría acabado constituyendo el contenido de un verdadero derecho de los ciudadanos. Que no quepa hablar de un derecho individual «a la» seguridad pública, se desprende por lo demás de las dificultades estructurales que afectan a la categorización de una tal situación jurídica subjetiva, no pudiendo sostenerse ni que se trate de un derecho ejercitable independientemente de las razones que lo justifiquen (pues semejante actitud arbitraria podría llevar a incurrir en delito a tenor de los arts. 392

* El presente trabajo tiene su base en la ponencia pronunciada en el Congreso sobre " $L a$ funzione della sicurezza nella legalità costituzionale" con ocasión del Bicentenario de la Fundación del Cuerpo de Carabineros, organizado por iniciativa del Consejo del Estado y de la Comandancia General del Cuerpo de Carabineros, celebrado en Roma, Palazzo Spada, el 16 de octubre de 2014. Traducción de la versión original italiana por Luis Jimena Quesada.

${ }^{1}$ En verdad, como ha subrayado Baumann, Z.: Dallo Stato sociale allo Stato di sicurezza, en Id., L'Europa è un avventura (Europe. An Unfinished Adventure, 2004), trad. it. M. Cupellaro, RomaBari, 2006, 109 ss., la percepción de la seguridad pública habría cambiado tras treinta años desde el fin de la Segunda Guerra Mundial. Para una confirmación de tal tesis, con relación a Italia, véase mi contribución Libertà e sicurezza. Cinquant'anni dopo, en Dir. e soc. 2013, 203 ss. 
y 393 del Código Penal italiano) al margen, consiguientemente, de los límites consentidos por la autodefensa (art. 52 del Código Penal italiano), ni que constituya un pretendido derecho a prestaciones positivas de la policía y de los carabineros mediante simple petición del ciudadano, sin ningún margen de discrecionalidad por parte de las fuerzas del orden.

Ilustrativo en tal dirección es el Auto n. ${ }^{\circ} 187$ de 2001 de la Corte Constitucional italiana, en el cual se puso de relieve la naturaleza contradictoria de la tesis defendida por el órgano jurisdiccional a quo, según el cual, entre los derechos humanos inviolables (art. 2 de la Constitución italiana, CI), se encontraría asimismo el derecho "a la protección de la propia seguridad frente a la comisión de bechos delictivos" mediante la intervención de la autoridad judicial limitativa de las demás libertades personales». Por el contrario, la Corte observó sobre este punto que «corresponde exclusivamente a la discreción del legislador la determinación, de conformidad con el principio de reserva de la ley establecido por el art. 13, párrafo 2, de la Constitución, de los casos en que el órgano jurisdiccional puede ordenar restricciones a la libertad personal, y queda igualmente reservada a la discrecionalidad del legislador la determinación de los casos excepcionales de necesidad y urgencia en los que pueden adoptarse medidas provisionales que limiten la libertad personal en el sentido del art. 13, párrafo 3, de la Constitución».

Precisamente, por ello, el título del presente Congreso alude a la clásica «función de seguridad», y no al derecho a la seguridad. No tanto porque al ejercicio de una función de seguridad no puedan corresponder pretensiones calificables como «derechos», cuanto porque la seguridad «pública» no puede, como acaba de subrayarse, constituir el contenido de un derecho de la persona o de un derecho social. La seguridad pública — que no debe ser confundida con la genérica necesidad de seguridad que atañe a los sectores más variados ${ }^{2}$, como la seguridad económica, la seguridad de la propia salud, etc.- constituye, en efecto, como se verá, un estado psicológico colectivo del que emana un interés difuso, cuya tutela es de competencia exclusiva del Estado.

\section{SOBRE QUÉ DEBE ENTENDERSE POR SEGURIDAD EN RELACIÓN CON EL ORDEN PÚBLICO}

Como es sabido, la introducción en el ordenamiento italiano de la locución «orden y seguridad pública» se produce a través del art. 2 del Texto Refundido de la Ley de Seguridad Pública (TRLSP) de 1926 (posteriormente reproducido

${ }^{2}$ Con respecto a ello, véase Ridola, P.: Libertà e diritti nello sviluppo storico del costituzionalismo, en R. Nania e P. Ridola (coord.), I diritti costituzionali, II ed., vol. I, Torino, 2006, 138 ss. 
en el TRLSP de 1931) ${ }^{3}$, en virtud de cual «el Prefecto, en caso de urgencia o de grave necesidad pública, tiene la facultad de adoptar las medidas indispensables para la tutela del orden y de la seguridad pública».

Aunque la Constitución italiana, hasta la reforma constitucional de 2001, haya hecho referencia solamente a la seguridad pública — no en cambio así dos de los cinco Estatutos regionales especiales ${ }^{4}$ — la doctrina, con la salvedad de algunas iniciales perplejidades ${ }^{5}$, ha considerado constitucionalmente legítimo que en las leyes ordinarias se haga mención al orden público. Ello no obstante, casi de manera unánime se ha considerado asimismo —en coherencia con los principios de la Constitución italiana que garantizan la circularidad del poder político y el disenso ideológico siquiera en su expresión «fuerte»- que el concepto de orden público designa meramente el orden material (el «ordre dans la rue» de Marcel Hauriou) $)^{6}$, el cual sólo puede considerarse perturbado como consecuencia de comportamientos humanos violentos o por hechos naturales (un terremoto, unas inundaciones) que puedan ser perjudiciales para la seguridad pública ${ }^{7}$.

3 Art. 2 del Real Decreto n. ${ }^{\circ} 1848$ de 6 de noviembre de 1926, reproducido en el todavía vigente art. 2 del TRLSP n. ${ }^{\circ} 773$ de 18 de junio de 1931.

${ }^{4}$ En efecto, aludían también al orden público el art. 31 del Estatuto de Sicilia (Ley constitucional n. ${ }^{\circ} 2$ d 26 de febrero de 1948) y el art. 44 del Estatuto del Valle de Aosta (Ley constitucional n. ${ }^{\circ} 4$ de 26 de febrero de 1948).

5 Barile, P.: Il soggetto privato nella Costituzione italiana, Padova, 1954, 117.

${ }^{6}$ Hariou, M.: Précis élémentaire de droit administratif, IV ${ }^{a}$ ed., Paris 1938, 34. El ilustre autor no contraponía el orden material al orden ideal, como en ésta y en otras de mis contribuciones, pero extraía del concepto material del orden que las autoridades administrativas sólo podrían afectar a las libertades públicas cuando su ejercicio hubiere determinado violaciones del orden material.

7 Puede verse, ya con anterioridad, en este sentido, PACE, A.: Il concetto di ordine pubblico nella Costituzione italiana, en Arch. giur., vol. CLXI, 1963, 111 ss.; Id., Libertà e sicurezza. cit., 192 ss. El planteamiento allí seguido, para diferenciar el orden público (material) del orden ideal difiere del enfoque secundado por L. Paladin, voz Ordine pubblico, en Noviss. Dig. it., vol. XII, Torino $1965, \S 3$, quien sin embargo análogamente distingue el orden público (material) del orden constitucional (que es ideal). Véase en tal dirección mi contribución Ordine pubblico, ordine pubblico costituzionale, ordine pubblico secondo la Corte costituzionale, en Giur. cost.,1971, 1777 ss. La tesis aquí planteada difiere menos de la sucesivamente sostenida por Lavagna, C.: Il concetto di ordine pubblico alla luce delle norme costituzionali, en Dem. dir. 1967, 367 ss., 372, quien, pese a partir de una concepción normativa del orden público, alcanza la misma conclusión acerca de la naturaleza esencialmente material del concepto de orden público en la Constitución italiana. Concretas referencias a la tesis que yo mantengo cabe encontrarlas en las excelentes contribuciones de A. CERRI, voz Ordine pubblico, en Enc. giur. it. 1990; Id., voz Ordine pubblico, en Enc. giur. it., Aggiornamento, ivi, vol. XXV, Roma 2007. Una acertada distinción de los dos conceptos se encuentra asimismo en Corso, G.: L'ordine pubblico, Bologna, 1979, 133 ss. 
Consiguientemente, la seguridad pública identifica el estado psicológico de la colectividad que se sienta segura en cuanto a su persona y sus bienes. Y es justamente en estos términos en los que se expresó la Corte Constitucional italiana en una de sus primeras sentencias, cuando afirmó que «se tiene seguridad cuando el ciudadano puede desarrollar sus actividades legítimas sin verse amenazado por ofensas a su personalidad física y moral; es "la vida civil ordenada", en la que radica indudablemente la meta de un Estado de Derecho, libre y democrático» ${ }^{8}$.

La neta distinción entre orden y seguridad esbozada por Mussolini en el informe ministerial al TRLSP de 1926 con relación al art. 2 - a tenor de la cual «la tutela del orden atañe más directamente a la defensa de los intereses objetivos del Estado, mientras que la tutela de la seguridad atañe más directamente a la defensa de los ciudadanos $y$ de sus bienes»— ofrecía una imagen deliberadamente edulcorada (¡sensible a la defensa de los ciudadanos y de sus bienes!), pero repárese en que, en primer lugar, el referido Texto Refundido estaba «inspirado en la autoridad del Estado y la celosa tutela de las prerrogativas de la soberanía inherente al poder del Gobierno» ${ }^{9}$ y, en segundo lugar, según el Ministro de Gracia y Justicia Alfredo Rocco, los derechos de los ciudadanos constituían, exhumando las ideas de Gerber ${ }^{10}$, únicamente «derechos reflejos» frente al poder del Estado ${ }^{11}$.

En realidad, tanto entonces como actualmente, seguridad y orden público son las dos caras de la misma moneda: en los regímenes autoritarios la seguridad pública es la seguridad «hipostasiada» del Estado-persona, en los ordenamientos democráticos es la seguridad «hipostasiada» del Estado-comunidad ${ }^{12}$. En otras palabras, en los ordenamientos democráticos (rectius, en los ordenamientos de democracia liberal) la seguridad pública existe en tanto en cuanto se mantenga el orden material. Y ello no sólo gracias a las funciones de seguridad pública sino,

${ }^{8}$ Sentencia n. ${ }^{\circ} 2$ de 1956.

9 Checchi, O.: Il nuovo codice di p.s.: commentato e preceduto da una nota illustrativa, Firenze, $1927,4$.

${ }^{10}$ Von Gerber, C. F.: Lineamenti del diritto pubblico tedesco (Grundzüge des deutschen Staatsrecht, 1880), trad. it. P. L. Lucchini, en Id., Diritto pubblico, Milano, 1971, que precisamente hablaba de los derechos del ciudadano como «efectos reflejos» del poder del Estado (ivi, 130 nota 45) o de «derechos reflejos», por cuanto el ciudadano «pertenece a dicho Estado como sujeto jurídico, como persona jurídica sometida» (ivi, 202). En la página sucesiva se encuentra la famosa formulación de los «derechos reflejos».

${ }^{11}$ Sobre este punto, véanse las duras críticas dirigidas a Alfredo Rocco por Ruffini, F.: Diritti di libertà, Torino, 1926, 102 ss., retomadas asimismo por P. Ungari en Alfredo Rocco e l'ideologia giuridica del fascismo, Brescia, 1963, 65 ss.

12 Sobre la evolución de los conceptos de policía (y de orden público) desde la Monarquía hasta el Estado democrático véase, por referencia al ordenamiento alemán, Denninger, E.: Polizei in der freiheitlichen Demokratie, Frankfurt am Main - Berlin, 1968. 
más aún, «con medidas de seguridad social y otros servicios públicos» aptas para eliminar al máximo las causas de potenciales desórdenes ${ }^{13}$.

Al contrario, en los ordenamientos en los que la circularidad del poder no queda en absoluto garantizada o la circularidad del poder se encuentra prevista pero bajo determinadas condiciones ${ }^{14}$, el orden público se identifica con un orden ideal con respecto al cual las posturas disidentes son consideradas como peligrosas para la seguridad del régimen. Hipótesis, ésta, que cabe hallar no sólo en los ordenamientos totalitarios (fascista, nacionalsocialista o comunista) en los cuales está incluso prohibida la disidencia pacífica, sino que se encuentra, si bien con diverso grado de intensidad, asimismo en algunos ordenamientos social-democráticos o incluso liberal-democráticos, cuando en ellos se niegue la libertad de quienes nieguen la libertad. Por el solo hecho de que en estos últimos pueda a veces negarse la libertad a quienes nieguen la libertad ${ }^{15}$, también ellos acaban de hecho siendo tributarios de un concepto ideal de orden público que, aunque no

13 Así, el conocido politólogo y constitucionalista inglés Sir Jennings, W. I.: Public Order, en Political Quarterly, vol. 8, 1937, 7 ss.

14 Véase, por ejemplo, el art. 18 de la Ley Fundamental de Bonn (GG), que prevé la privación de toda una serie de derechos fundamentales cuando éstos sean ejercidos «para luchar contra el orden fundamental liberal-democrático» de la República Federal Alemana. De ahí, como es conocido, la calificación de la democracia alemana, por parte del Tribunal Constitucional Federal, como «streitbar» (BVerfGE, 5, 85, 134 ss.), concepto al cual el Tribunal Constitucional se ha mantenido fiel asimismo en sentencias posteriores (véase KreBs, W.: Art. 18, en Von MüNCH, I.-KüNING, P.: Grundgesetz-Kommentar, vol. I, V ed., München, 2000, 1026), según el planteamiento conceptual de Löwenstein, K.: Militant Democracy and Fundamental Rights, in Columbia Law Review, vol. XXXVIII, 1938, 417 ss., 638 ss.

15 Sintomática resulta la posición sostenida al respecto por un estudioso de orientación liberal como A. C. Jemolo (del cual, véase I problemi pratici della libertà, Milano 1961, 51 ss. y 124 ss., para el planteamiento teórico y para la invitación a resolver el problema caso por caso, y el comentario de sentencia Lo Stato può difendersi, en Giur. cost. 1970, 957 ss., en el cual el ilustre autor efectuó un comentario de adhesión a la sentencia interpretativa de desestimación o rechazo n. ${ }^{\circ} 65$ de 1970 de la Corte Constitucional italiana relativa al art. 414 del Código Penal en materia de apología e incitación al delito). En el mismo sentido, véase BognetTi, G.: Apologia di delitto e principi costituzionali di libertà d'espressione, en Riv. it. dir. proc. pen. 1960, 195 ss.; Id., Apologia di delitto punibile ai sensi della Costituzione e interpretazione della norma dell'art. 414, ultimo comma, cod. pen., ivi 1971, 18 ss.; Id., Il pericolo nell'istigazione all'odio di classe e nei reati contro l'ordine pubblico, en Giur. cost. 1974, 1441 ss., quien considera oportuna «dejar al legislador la posibilidad, para situaciones más o menos excepcionales, de introducir medidas de control de discursos incendiarios que, a su juicio, parezcan peligrosos» $\mathrm{y}$, por ende, se pronuncia a favor de la constitucionalidad del delito de apología considerando suficiente la peligrosidad no inmediata. Para una crítica a tales tesis, véase M. ManetTi, in Pace, A., y Manetti, M.: Art. 21. La libertà di manifestazione del proprio pensiero, en Commentario della Costituzione a cura di G. Branca e A. Pizzorusso, Roma-Bologna, 2006, 241. 
revista un carácter tan acechador como el de los ordenamientos totalitarios, ciertamente es un orden que pretende algo más que el mero «ordre dans la rue». En efecto, no puede calificarse como «liberal» sin otros aditamentos el ordenamiento que, pese a revestirse con formas de enmascaramiento, no tolera la disidencia.

Ahora bien, ya que es contradictorio tanto fundamentar la libertad en la negación de la disidencia radical, como admitir la disidencia radical al tiempo que se reprimen los comportamientos que la manifiesten, así como consentir que la disidencia radical pueda doblegar la voluntad de los electores ${ }^{16}$, la única vía razonable para salir de semejante encrucijada, sin dejar de garantizar la posibilidad de disentir, consiste en prohibir la disidencia radical sólo cuando la apología de los comportamientos potencialmente subversivos del orden democrático disponga de la concreta capacidad de provocar la inmediata perpetración de delitos. Y esto es lo que la Corte de Casación italiana ha subrayado en al menos un par de ocasiones ${ }^{17}$.

\section{LOS LÍMITES IMPUESTOS A LA FUNCIÓN DE SEGURIDAD DE LA LEGALIDAD CONSTITUCIONAL}

Contrariamente a lo mantenido por algunos estudiosos autorizados, el principio de legalidad, en lo que respecta a la actividad administrativa encuentra también, pese a que no exclusivamente, en la Constitución sus bases normativas: en primer lugar, en el art. $97 \mathrm{CI}$, que impone a todos los empleados públicos que se muevan en el ámbito predeterminado por sus «esferas de competencia» (principio de legalidad formal); en segundo lugar, en el artículo 23 CI, que establece que la imposición de una obligación debe ir respaldada por una base legal previa, lo que vale asimismo para la imposición de una prohibición (dado que la «prohibición» no es otra cosa que una «obligación de no hacer») ${ }^{18}$; por último, en el art. 113 CI, el cual, al disponer que contra los actos de la Administración Pública cabe siempre la tutela jurisdiccional de los derechos e intereses, impone que cualquier medida administrativa debe poder ser contrastada con un supuesto previamente regulado (principio de legalidad sustancial). En este sentido, la

16 Cerri, A.: voz Ordine pubblico, cit., 3.

17 Así, las Sentencias de la Sala de lo Penal de la Corte de Casación (Cass., sez. I pen.) de 17 de noviembre de 1997, n. ${ }^{\circ} 11578$; Id., 1 de junio de 2001, n. ${ }^{\circ} 26907$, en las cuales se han considerado punible la apología de un delito cuando la acción tenga «la concreta capacidad de provocar la inmediata ejecución de delitos o, cuando menos, la probabilidad de que tales delitos sean cometidos en un futuro más o menos próximo».

18 En este sentido, con ulteriores indicaciones favorables, véase Gavazzi, G.: L'onere. Tra la libertà e l'obbligo, Torino, 1970, 51 nota 25. 
Corte Constitucional ha subrayado en numerosas ocasiones el obligado respeto de los denominados principios de legalidad «formal»y «sustancial» resaltando que «La "absoluta indeterminación del poder atribuido a una Administración Pública sin criterio alguno por parte de la ley" vulnera el principio de legalidad sustancial»; y que «No es suficiente que el poder venga condicionado en la ley a la tutela de un determinado bien o de un valor, sino que resulta indispensable que su ejercicio venga determinado en cuanto al contenido y a las modalidades de tal manera que se mantenga constantemente una, aun elástica, cobertura legislativa de la acción administrativa» ${ }^{19}$.

A ello se añade que el principio de legalidad, en Derecho Administrativo, comporta además el respeto de los principios de tipicidad, de nominatividad y de proporcionalidad. Principios que, en línea de principio, obviamente se aplican asimismo a las medidas tendentes al mantenimiento del orden y de la seguridad pública.

Expresado lo cual, no me detendré a enumerar las normas legales, por lo demás de todos conocidas, que atribuyen la competencia del mantenimiento del orden público y de la seguridad pública al Ministerio del Interior y a los servicios de él dependientes, así como a las fuerzas de policía dependientes de otras administraciones; como tampoco me parece que venga al caso el tener que subrayar la clara mayor determinación del concepto de orden y de seguridad pública en el modo enunciado por el art. 159, párrafo 2, del Decreto Legislativo n. ${ }^{\circ} 112$ de $1988^{20}$, en comparación con la deliberada indeterminación del art. 2 del TRLSP de $1931^{21}$.

Merece la pena en cambio resaltar que sí pueden ser adoptadas medidas de policía por motivos de orden y de seguridad pública dirigidas a limitar derechos constitucionalmente previstos, siempre que la Constitución lo consienta expresamente o bien lo reserve asimismo de manera explícita a la ley ordinaria. Por añadidura, tanto en el caso de que la posible limitación se encuentre prevista por

19 Cassese, S.: Il diritto amministrativo e i suoi principi, en Id. (coord.), Istituzioni di diritto amministrativo, IV ed., Milano, 2012, 12, que cita las sentencias n. ${ }^{\circ} 31$ de 2009 y n. ${ }^{\circ} 115$ de 2011 de la Corte Constitucional italiana.

${ }^{20} \mathrm{El}$ art. 159, párrafo 2 del Decreto Legislativo n. ${ }^{\circ} 112$ de 31 de marzo de 1998 dispone que las «funciones y los deberes relativos al orden y a la seguridad pública a los que se refiere el art. 1, párrafo 3, letra e) de la Ley n. ${ }^{\circ} 59$ de 15 de marzo de 1997, atañen a las medidas preventivas y represivas dirigidas al mantenimiento del orden público entendido como el complejo de los bienes jurídicos fundamentales y de los intereses públicos primarios sobre los cuales se basa la convivencia ordenada y civil en la comunidad nacional, así como a la seguridad de las instituciones, de los ciudadanos y de sus bienes».

21 ... merced al cual continuó siendo aplicado en sentido inconstitucional incluso tras la Sentencia n. ${ }^{\circ} 26$ de 1961 de la Corte Constitucional. Para indicaciones en tal sentido, véase PACE, A.: Libertà individuali e qualità della vita, Napoli, 2008, $17 \mathrm{~s}$. 
la Constitución, como en el supuesto de que dicha limitación quede reservada a ley, la posibilidad de medidas preventivas para la tutela del orden y de la seguridad pública debe estar, ella también, expresa y específicamente prevista. Lo cual deja desprovisto de todo fundamento a la doctrina favorable a un hipotético poder general de prevención ${ }^{22}$.

Señalado lo anterior, pasaré a referirme brevemente a las disposiciones constitucionales que prevén intervenciones —en algunos casos incluso de carácter preventivo — por motivos de orden y de seguridad pública.

En primer lugar, no resulta controvertido que dichas intervenciones se hallen pacíficamente previstas como limitación de la libertad de circulación (art. 16, párrafo $1 \mathrm{CI}$ ), de la libertad de reunión (art. $17 \mathrm{CI}$ ) incluso a título preventivo («por motivos contrastados», art. 17, párrafo $3 \mathrm{CI}$ ) y de la libertad de iniciativa económica privada (art. 41, párrafo $2 \mathrm{CI})^{23}$.

En segundo lugar, pueden asimismo ser adoptados motivos de orden y de seguridad pública con relación a la libertad personal. El art. 13, párrafo $3 \mathrm{CI}$ permite efectivamente restricciones «provisionales» a tal libertad «en casos excepcionales de necesidad y de urgencia, especificados taxativamente en la ley». Por consiguiente, si se considera, como parece correcto entender, que la reserva de ley prevista en dicha disposición no sea reforzada pese a ser absoluta, no cabe sostener que las restricciones disciplinadas por dicho precepto sean solamente aquellas que «sirvan» a los fines de la justicia penal, de educación de menores o de tutela de la salud ${ }^{24}$.

22 En el sentido criticado, véase IsEnseE, J.: Das Grundrecht auf Sicherbeit. Zu den Schutzpflichten des Freibeitlichen Verfassungsstaates, Berlin-New York 1983; Cerrina Ferroni, G., y MorBIDELLI, G.: La sicurezza come valore superprimario, en Percorsi costituzionali, n. 1, 2008, 31 ss., 41. En sentido contrario, en cambio, véanse las insuperables observaciones de DenNinger, E.: Diritti dell'uomo e Legge fondamentale (Menschenrechte und Grundgestetz, 1994), trad. it. C. Amirante, Torino 1998, 38, 89.

${ }^{23}$ Por lo demás, se encuentra difundida en la doctrina la consideración según la cual la seguridad, en el art. 41, presenta un doble significado: como seguridad pública y como seguridad de los trabajadores.

${ }^{24}$ En sentido contrario, véase Elia, L.: Libertà personale e misure di prevenzione, Milano, 1962, 23 ss.; Id., Le misure di prevenzione tra l'art. 13 e l'art. 26 della Costituzione, en Giur. cost., 1964, 949 ss., así como, más recientemente, entre otros muchos, FiLippetTA, G.: La libertà personale, la libertà di domicilio, di circolazione e individuale, en NANIA, R., y RIDOLA, P.: I diritti costituzionali, cit., vol. II, 563, con indicaciones sobre los autores favorables y contrarios. También es cierto, según los críticos, que la tesis aquí mantenida implicaría en abstracto un vacío en los fines de las actividades de policía. Sin embargo, no es menos cierto que, tratándose de una materia cubierta por la reserva absoluta, es deber del legislador precisar en cada ocasión los fines y especificar los casos y las modalidades que ha de revestir la intervención de las autoridades competentes en materia de seguridad pública. 
De ahí la legitimidad de las restricciones a la libertad personal por motivos de seguridad pública, ya sean «indefinidas» o simplemente «temporales» ${ }^{25}$, siempre que la ley indique expresamente los casos excepcionales en los que la restricción pueda ser efectuada y siempre que la ley prevea su control oportuno por parte de la autoridad judicial.

Lo cual viene a significar que la disciplina constitucional del art. 13, párrafos 2 y 3 CI ahora se aplica pacíficamente también a las restricciones que no sean al tiempo perjudiciales para la dignidad social ${ }^{26}$ — como en cambio estuvo durante un tiempo sosteniendo la Corte Constitucional con aberrantes consecuencias respecto de la exclusión de las restricciones a la libertad personal por motivos de salud $^{27}$. En esta línea, más acorde con los dictados constitucionales, han sido por lo demás progresivamente eliminadas las hipótesis de acompañamiento coercitivo sustraídas al control judicial sucesivo ${ }^{28}$. Al contrario, la disciplina del registro o cacheo por motivos de orden público ${ }^{29}$ — que correctamente prevé el control judicial — suscita reservas de otro tipo, puesto que la ley, más que especificar los

${ }^{25}$ En el sentido de que las restricciones disciplinadas por el art. 13 CI serían solamente las de carácter indefinido, y no las de naturaleza temporal, como el acompañamiento coercitivo de seguridad pública disciplinado por el art. 15 del TRLSP de 1931. Véase Corte Constitucional, Sentencia n. ${ }^{\circ} 13$ de 1972, en Giur. cost., 1972, 70 ss., con observaciones críticas de PACE, A.: La negata incostituzionalità dell'accompagnamento coattivo di pubblica sicurezza. En sentido crítico, sobre este punto, véase asimismo FiLippetta, G.: La libertà personale, cit., 582.

26 ... como algunas medidas de identificación (art. 4, TRLSP). Sobre tal punto, véase Corte Constitucional, Sentencia n. ${ }^{\circ} 30$ de 1962. En sentido igualmente crítico, CARETTI, P.: La libertà personale, en el volumen Libertà costituzionali e limiti amministrativi, en el Trattato di diritto amministrativo (G. Santaniello, dir.), Padova, 1990, 29 s.; Id., voz Libertà personale, en Disc. pubbl., vol. IX, Torino, 1994, 238 s. No parece, en cambio, que la Corte Constitucional se haya hecho eco de la tesis crítica cuando, en la Sentencia n. ${ }^{\circ} 105$ de 2001, vino a afirmar que «cualquier circunstancia de sometimiento físico al poder de otro» determina «la humillación de la dignidad humana», que es «un índice seguro de la afectación de la medida a la esfera de la libertad personal».

27 Véase sobre este aspecto Piccione, D.: La crisi della giurisdizione sui provvedimenti limitativi della libertà personale estranei a fini penali, en Giur. cost., 2002, 2157 ss.

${ }^{28}$ En este sentido, el art. 349, párrafo 5, del Código de Procedimiento Penal, prevé que la policía judicial comunique inmediatamente al Ministerio Público la detención, a efectos de identificación, de una persona en sus dependencias, incluso aunque la detención se demore menos de doce horas. Véase, sin embargo, la Sentencia n. ${ }^{\circ} 105$ de 2001 de la Corte Constitucional en Giur. cost., 2001, 1680 ss. con comentarios de BASCHERINI, G.: Accompagnamento alla frontiera e trattenimento nei centri di permanenza temporanea: la Corte fra libertà personale e controllo delliemigrazione, así como de Piccione, D.: Accompagnamento coattivo e trattenimento dello straniero al vaglio della Corte costituzionale: i molti dubbi su una pronuncia interlocutoria. Véanse en cambio asimismo las posteriores Sentencias n. ${ }^{\circ} 106$ de 2002 y 222 de 2004, que el Legislador ha secundado con la Ley n. ${ }^{\circ} 271$ de 12 de noviembre de 2004.

29 Art. 4 de la Ley n. ${ }^{\circ} 152$ de 22 de mayo de 1975. 
«casos de necesidad y de urgencia», se limita a reproducir servilmente el enunciado constitucional, vulnerando con ello la ratio del precepto constitucional.

Diversamente, no pueden ser establecidas limitaciones a la seguridad pública en relación con la libertad domiciliaria, la libertad de correspondencia y comunicación, la libertad de asociación, la libertad de manifestación del pensamiento o la libertad de prensa y radiodifusión radiotelevisiva.

De igual modo, motivos de orden y de seguridad pública no pueden ser invocados con respecto a las inspecciones y los registros domiciliarios (art. 14 CI), por la sencilla razón de que, tratándose de un lugar privado, no cabe admitir como relevantes motivos de orden y seguridad «pública». Las inspecciones y los registros domiciliarios pueden ser en cambio ordenados cuando medie un motivo fundado para estimar que en el domicilio se haya cometido un delito ${ }^{30}$. Lo que resulta predicable asimismo de las denominadas inspecciones ambientales ex art. 266, párrafo 2, del Código de Procedimiento Penal ${ }^{31}$.

La limitación de la libertad de correspondencia y de comunicación, a tenor del precepto constitucional (art. 15 CI), puede ser ordenada «solamente por auto motivado de la autoridad judicial con las garantías establecidas por la ley». Lo cual, ya de por sí, excluye la posibilidad de incidir en tales libertades con medidas de la autoridad de seguridad pública. Y, en efecto, para el secuestro de la correspondencia procede materialmente un oficial de "policía judicial», que deberá entregarla inmediatamente a la autoridad judicial sin abrirla o alterarla.

La adopción de medidas de seguridad pública no se encuentra ni siquiera prevista con relación a la libertad de asociación. En verdad, si el límite al derecho de asociación viene identificado en la Constitución (art. 18 CI) con respecto a los «fines que no estén prohibidos a los individuos por la ley penal», ello quiere decir que se intentaba eliminar la posibilidad de esos poderes administrativos discrecionales que anteriormente aparecían caracterizados en el TRLSP. Lo cual viene confirmado por la Ley n. ${ }^{\circ} 645$ de 1952 e la Ley n 17 de 1982, relativas respec-

30 Art. 247 y siguientes del Código de Procedimiento Penal.

31 Sobre esta cuestión, véase la Sentencia n. ${ }^{\circ} 135$ de 2002 de la Corte Constitucional, en Giur. cost., 2002, 1062, ss. con comentarios de PACE, A.: Le videoregistrazioni ambientali tra gli artt. 14 e 15 Cost., así como de MARINI, F. S.: La costituzionalità delle riprese visive nel domicilio: ispezione o libertà «sotto-ordinata»? La Corte Constitucional, pese a rechazar la cuestión de legitimidad constitucional del art. 266, párrafo 2 del Código de Procedimiento Penal al contrastarlo con el canon de los arts. 3 y $14 \mathrm{CI}$, acertadamente afirmó en dicha decisión que los concretos conceptos expresados con la locución «inspecciones, registros o incautaciones» (párrafo 2) o con la locución «comprobaciones e inspecciones» (párrafo 3) no pueden, aunque sea por finalidades garantistas, quedar «cristalizados» en el tiempo. Consiguientemente, la Corte consideró constitucionalmente admisibles las denominadas inspecciones ocultas, esto es, la captación de imágenes de la vida privada mediante videocámaras ambientales, por lo demás sometidas a control judicial. 
tivamente a las asociaciones neofascistas y a las secretas, cuya disolución (y correspondiente confiscación de sus bienes) compete al Gobierno tras la previa resolución jurisdiccional mediante la que se determine el carácter ilícito penal de la asociación ${ }^{32}$.

Por último, teniendo en cuenta la dimensión meramente material del concepto de orden y seguridad pública, ninguna medida de policía de seguridad podría ser adoptada como limitación a la libertad de expresión, cualquiera que sea el medio de difusión. Ello se desprende de los principios de pluralismo sobre los que se fundamenta nuestra Constitución, pero se comprueba asimismo en el párrafo tercero del art. 21 CI. En su versión definitiva, el párrafo atribuye, en los casos específicamente previstos, el poder de secuestro de la prensa a la autoridad judicial y, en los casos de absoluta urgencia y con respecto a la prensa periódica, a los funcionarios de la "policía judicial» ${ }^{33}$. Lo cual deja desprovista de fundamento a la posibilidad de una intervención para proteger la seguridad y el orden público.

Title:

THE SAFETY FUNCTION UNDER THE CONSTITUTIONAL LEGALITY

\section{Summary:}

I. Why is it wrong and confusing to refer to a Right to Security. II. What should be understood by Public Order in relation to Public Safety either in an Authoritarian Government or in a Democratic one. III. The limits imposed to the Safety Function under the Constitutional Legality.

\section{Resumen:}

El presente artículo reflexiona acerca de las nuevas dimensiones en el planteamiento de la seguridad pública por el terrorismo y el extremismo. Desde esta perspectiva, el autor critica en primer término la tendencia a utilizar de modo impropio y confuso el término «derecho a la seguridad» por cuanto la seguridad es el contenido de una función del Estado que no se resuelve en un derecho individual; en segundo

32 Para ulteriores desarrollos sobre este punto, permítaseme remitir a PACE, A.: Problematica delle libertà costituzionali. Parte speciale, Padova, 1992, 374 ss.

33 Sobre esta cuestión, véase M. Manetti, in Pace, A., y Manetri, M.: Art. 21, cit., 774, en donde se recuerda que fue mérito de la Comisión de coordinación el haber optado por «policía judicial» en lugar de «policía de seguridad», pese a que esta última locución hubiere podido encontrar en el pleno un amplio consenso. 
lugar, el autor confirma su tesis, ya sostenida hace más de cincuenta años, según la cual, de un lado seguridad pública y orden público son las dos caras de la misma moneda y, de otro lado, el concepto de orden público asume un significado diverso según el contexto políticoinstitucional: «orden material» en los ordenamientos democráticos, el cual puede quedar lesionado sólo por comportamientos violentos o por hechos naturales (terremotos, inundaciones, etc.); «orden ideal» en los ordenamientos autoritarios en los que el orden público puede quedar dañado asimismo por comportamientos pacíficos como una manifestación pública del pensamiento; y finalmente aborda la extensión de los límites impuestos a la función de seguridad en la legalidad constitucional.

\begin{abstract}
:
The essay underlines that, even in the light of the current global challenges set out by terrorism and extremism, public safety is not a per se individual right but only the aim of a public function of the State. In this sense, the author holds that public order has a different meaning if taken into account in a democratic government or in a totalitarian one: in the first one, public order depicts a situation of «material» peace (the absence of disorder caused by human or natural events); in the latter, public order depicts the absence of «ideal» disorder caused even by pacific behaviours. He finally focuses on the extent of the limits to the public function of the State in this specific field both in general and in relation to the single constitutional rights.
\end{abstract}

\title{
Palabras clave:
}

Seguridad pública, orden público, orden constitucional, derecho individual, interés difuso o colectivo, margen de discreción, acción administrativa, reserva de ley, control judicial, democracia y disidencia.

\section{Keywords:}

Public Safety, Public Order, Constitutional Order, Individual Right, Collective Interest, margin of discretion, administrative action, legal reserve, judicial control, democracy and dissent. 